\title{
Essai de modélisation des flux solides dans l'Isère à Grenoble Relations avec l'hydroclimatologie
}

\author{
Solid discharge modelling for the Isère river near Grenoble \\ Relations with hydroclimatology
}

par A. Poirel

EDF/DTG Service Ressource en Eau, Grenoble

et

LTHE, Grenoble

Laboratoire des Transferts en Hydrologie et Environnement

Suspended solids discharge (SSD) was measured for two years on the Isère river near Grenoble (France). The relations between SSD and water discharge are complex because of sediment storage in the river channel bed and banks. Rainfall and snowmelt runoff are statistically identified as the major factors contributing to sediment yield. Water level in the main tributary (Arc river) is the major factor contributing to sediment removal from the channel system and to downstream sediment transport.

A sediment storage model was tested on this river, in order to simulate SSD. It is based on erosivity of liquid precipitation and snowmelt on the one hand, and sediment store removal by the river flow on the other hand. The aims of the model are to extend short time series of suspended sediment concentrations with hydrological variables, to adapt suspended sediment sampling with real time predictions and to help in management of sedimentation in the downstream reservoir of St Egreve.

The conclusions of the study are that the model can simulate the SSD, that the snowmelt is the major process to take into account in SSD on the Isère river and that the performance of the model calibration is limited by anthropic activity (upstream reservoir release and hydraulic scouring of upstream reservoirs under flood conditions).

\section{INTRODUCTION}

Le gestionnaire d'ouvrages hydrauliques qu'est Electricité de France est confronté au problème de la sédimentation dans les retenues particulièrement dans les retenues au fil de l'eau sur les grands cours d'eau alpins [1].

Si les dépôts des fortes granulométries en queue des retenues ont fait l'objet de nombreuses études dès la création des retenues, le problème majeur s'est vite révélé être le transport solide en suspension vu les tonnages mis en jeu. Celui-ci se dépose tout au long de la retenue et notamment en amont immédiat du barrage, pouvant envaser les vannes de fond et donc atteindre à la sûreté de l'ouvrage [2].
De plus, ce volume sédimentaire qui réduit la capacité de stockage de la retenue, peut poser des problèmes de stabilité de l'ouvrage et générer des nuisances sur l'environnement.

\section{II 口 PRÉSENTATION DU PROBLÈME}

2.1 La problématique de gestion du stock sédimentaire

Le gestionnaire d'ouvrages est confronté à une gestion des stocks sédimentaires fins qui transitent ou se stockent dans les retenues [3]. Cette gestion s'effectue principalement au travers de chasses hydrauliques mais également au travers d'opérations de dévasages, délimonages ou dragages beaucoup plus coûteuses. 
La gestion du stock de sédiments fins est particulièrement difficile car :

- les entrées dans le stock dépendent des flux de MES qui sont très variables en amplitude et très localisés dans le temps,

- les variations de stocks ne sont connues qu'à travers des bathymétries successives (imprécision élevée, évaluation annuelle au mieux...),

- les sorties du stock dépendent fortement des conditions hydrologiques, les chasses étant réalisées sur des hautes eaux (donc souvent sur des épisodes de fort transport solide en suspension) afin de minimiser les impacts sur l'environnement.

L'efficacité d'une chasse hydraulique est évaluée comme la résultante de deux termes, le premier étant la quantité de sédiments évacués de la retenue, le second étant la quantité de sédiments non déposés du fait de l'effacement du barrage (concept des « transparences »).

\subsection{Les outils du gestionnaire}

Les outils du gestionnaire en matière de sédimentation dans les retenues sont de deux ordres aujourd'hui :

- les modèles de transfert qui permettent ou de prévoir le dépôt des sédiments connaissant les concentrations en entrée de la retenue ou d'évaluer le transfert aval des MES connaissant les concentrations en sortie de la retenue lors des chasses ou vidanges,

- les modèles de reprise qui permettent de prévoir globalement l'efficacité d'une chasse sur le stock sédimentaire en place sachant les caractéristiques géométriques et rhéologiques de ce stock et les conditions hydrauliques [4]

\subsection{La demande actuelle}

Il manque donc au gestionnaire un outil d'évaluation du régime de transport solide en suspension qui lui permette de simuler une gestion sédimentaire de la retenue.

Pour les retenues existantes, il souhaite disposer d'une estimation du cumul des flux de MES sur une période donnée et d'une anticipation sur les épisodes de forts apports en MES. Pour les projets de retenues, il lui est nécessaire d'estimer des chroniques de MES réalistes à mettre en entrée des modèles de transfert.

Si la mesure des débits et des MES est une réponse logique à ce problème, la chronique nécessaire à l'estimation d'un régime de transport solide est très longue et très coûteuse à acquérir - environ 30 ans d'après Meybeck pour avoir une bonne précision sur le régime de flux en suspension.

Le gestionnaire qui souhaite une réponse plus rapide demande donc d'estimer les flux solides à partir des informations disponibles. Une réponse classique à cette demande consiste à estimer une relation statistique entre les débits généralement connus sur des périodes assez longues et les MES mesurées sur une courte période. Si cette relation est généralement satisfaisante pour les zones de production du débit liquide et du débit solide, il en va tout autrement à l'échelle de grands bassins versants. Cette relation est perturbée du fait de stockages intermédiaires de sédiments dans le lit de la rivière, du fait des hétérogénéités des différents sous-bassins (nature des terrains et régime des précipitations) et du fait de l'influence anthropique sur le bassin versant (modification des lits de rivières, modification du régime hydrologique, chasses et vidanges...).

Cette préoccupation recoupe deux autres problèmes actuels qui sont :
- l'estimation des flux polluants à l'échelle de grands bassins versants pour lesquels Meybeck a clairement montré que la connaissance des flux de MES était fondamentale,

- l'estimation de l'impact des fortes concentrations de MES en tant que «toxique» sur des populations piscicoles d'écosystèmes où les zones latérales de refuges se sont bien souvent raréfiées (endiguement) ou sont devenues difficilement accessibles (affluents 'perchés' par incision des lits).

\subsection{Objectifs du modèle proposé}

Le premier objectif du modèle proposé est d'estimer le flux sédimentaire journalier de l'Isère à Grenoble uniquement en fonction des données hydroclimatologiques disponibles sur le bassin versant. Il sera calé sur les chroniques de flux à Grenoble.

Le second objectif est de disposer en temps réel différé d'une estimation des flux entrés dans la chaîne d'ouvrages de la Basse Isère depuis la dernière chasse et d'une prévision des apports solides en suspension pour le lendemain. Ces deux informations permettent au gestionnaire, avec la prévision de l'hydrologie, d'estimer l'intérêt économique et environnemental de réaliser une chasse.

Enfin le troisième objectif sera à terme de déterminer une série reconstituée des flux à Grenoble permettant de proposer après simulation une gestion plus rationnelle des retenues de l'Isère en aval de Grenoble. Cela devrait permettre de proposer soit des abaissements partiels de plan d'eau limitant la décantation lors des forts apports de MES soit des chasses lorsque le cumul des dépôts est important. Notamment l'exploitant pourrait disposer d'un critère de décision pour mieux gérer les épisodes de crues successives.

\section{III — PRÉSENTATION DU SITE D'ÉTUDE ET DES DONNÉES DISPONIBLES}

\subsection{Le site d'étude}

La retenue de St Egrève est située en aval de Grenoble au confluent du Drac et de l'Isère. Sa capacité est de $6,8 \mathrm{hm}^{3}$, sa longueur de $6 \mathrm{~km}$ pour une largeur au barrage de $177 \mathrm{~m}$ et une profondeur maximale de 5 à $6 \mathrm{~m}$.

Par sa situation la retenue de St Egrève collecte le TSS issu de l'Arc moyen et Supérieur, en partie de l'Isère (aval de Tignes), de l'Arly et de la Romanche affluent du Drac (aval du Chambon).

La gestion des sédiments à St Egrève se fait par des chasses sur crues $\left(\mathrm{Q}>600 \mathrm{~m}^{3} / \mathrm{s}\right)$. Le tableau 1 donne les tonnages évacués de la retenue ainsi que les tonnages non déposés grâce aux chasses et à la première vidange (3/1992).

L'année 94 est très intéressante car 2 chasses ont été réalisées à 1 mois d'intervalle dans des conditions hydrologiques assez semblables mais très différentes en transport solide : l'intérêt de la seconde chasse est grandement majoré par le non-dépôt.

\subsection{Les données disponibles}

Les séries de mesures disponibles sont :

- les mesures de MES (Institut de Géographie Alpine) et de Hauteur d'eau avec la courbe de tarage associée (ENS d'Hydraulique et de Mécanique de Grenoble + EDF) sur la station de Grenoble Campus,

- les mesures de hauteur d'eau avec la courbe de tarage associée pour la station de Pont de St Rémy sur l'Arc inférieur (EDF), 


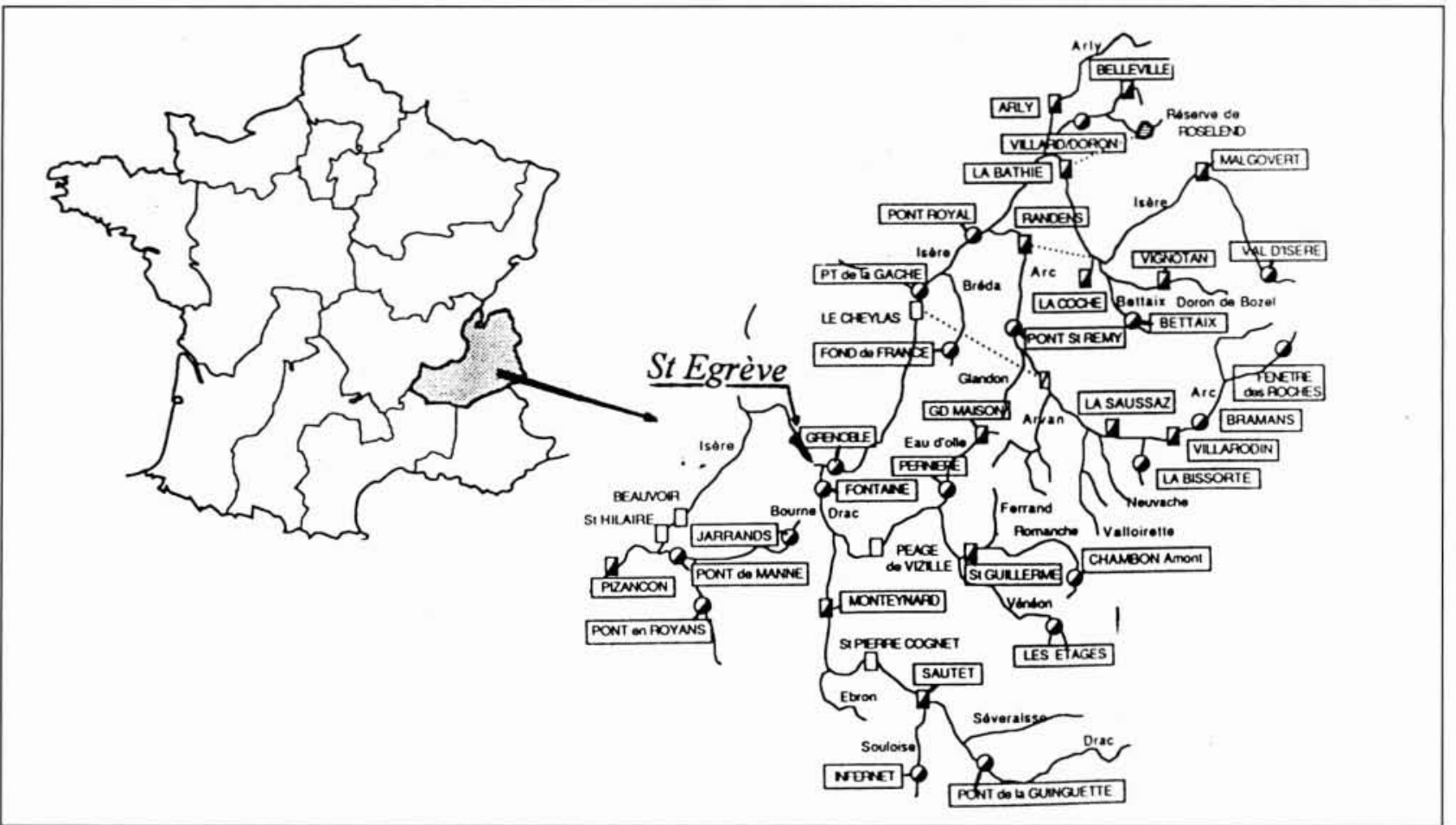

1. Localisation géographique de la zone d'étude.

Tableau 1. - Tonnages évacués de la retenue de St Egrève.

\begin{tabular}{|c|c|c|c|c|}
\hline Date & $\begin{array}{c}\text { Débit moyen journalier } \\
\text { à St Gervais }\end{array}$ & $\begin{array}{c}\text { Erosion } \\
\text { dans la retenue }\end{array}$ & Non-dépôt estimé & Tonnage efficace \\
\hline $15 / 3 / 1992$ & $282 \mathrm{~m}^{3} / \mathrm{s}$ & $180000 \mathrm{t}$ & $0 \mathrm{t}$ & $180000 \mathrm{t}$ \\
$29 / 10 / 1992$ & $863 \mathrm{~m}^{3} / \mathrm{s}$ & $500000 \mathrm{t}$ & $50000 \mathrm{t}$ & $550000 \mathrm{t}$ \\
$19 / 05 / 1994$ & $820 \mathrm{~m}^{3} / \mathrm{s}$ & $730000 \mathrm{t}$ & $25000 \mathrm{t}$ & $750000 \mathrm{t}$ \\
$27 / 06 / 1994$ & $884 \mathrm{~m}^{3} / \mathrm{s}$ & $230000 \mathrm{t}$ & $175000 \mathrm{t}$ & $400000 \mathrm{t}$ \\
$02 / 06 / 1995$ & $1039 \mathrm{~m}^{3} / \mathrm{s}$ & $600000 \mathrm{t}$ & $50000 \mathrm{t}$ & $650000 \mathrm{t}$ \\
\hline
\end{tabular}

- les mesures des pluviographes chauffants, et des thermographes gérés par EDF,

- les mesures de valeur en eau du manteau neigeux avec les télénivomètres EDF sur les bassins versants de l'Arc et de l'Isère.

Les différentes chroniques sont disponibles de manière concomitante depuis le 1/4/1994, date de mise en service des mesures de MES à Grenoble.

\subsection{Analyse des données}

L'analyse des chroniques de MES a été réalisée par Peyri (1996) qui a montré la faible corrélation avec les débits de l'Isère (environ $30 \%$ de variance expliquée).
Le tableau 2 donne pour différentes variables la moyenne par périodes sur la totalité de la chronique (1/4/94 au 30/9/96).

Il montre, que ce soit sur les concentrations ou les débits, l'influence prépondérante de la fusion nivale. Cela se traduit par un flux moyen 10 fois plus élevé au printemps qu'en hiver. On observe également que les concentrations moyennes en hiver sont beaucoup plus faibles qu'en été alors que les débits sont voisins. Cela s'explique par l'érosivité (cf. 4.1) des précipitations plus élevées en été.

Le LAMA a également mené [5] une étude sur l'érodabilité des terrains sur les sous-bassins d'ordre 4 de l'Isère à Grenoble. Cette étude a porté sur une variable géologique, une variable de couverture, la densité de drainage (longueur totale du réseau/surface du $\mathrm{BV}$ ) et le ratio de relief (dénive-

Tableau 2. -Moyennes de certaines variables par périodes.

\begin{tabular}{|l|c|c|c|c|c|}
\hline & & & & & \\
Moyenne & Erosivité $\mathrm{mm}^{2} / \mathrm{j}$ & Fusion $\mathrm{mm} / \mathrm{j}$ & MES Grenoble $\mathrm{mg} / \mathrm{l}$ & Q Grenoble $\mathrm{m}^{3} / \mathrm{s}$ & Flux $\mathrm{t} / \mathrm{s}$ \\
\hline Jan à Mar & 0,03 & 1,3 & 116 & 181 & 0,034 \\
Avr à Juin & 1,39 & $\mathbf{1 0 , 1}$ & $\mathbf{8 4 6}$ & $\mathbf{2 6 6}$ & $\mathbf{0 , 3 3 2}$ \\
Juil à Août & $\mathbf{5 , 6 8}$ & 2,4 & 444 & 199 & 0,121 \\
Sept à Déc & 0,98 & 0,7 & 307 & 147 & 0,061 \\
\hline
\end{tabular}


lée/longueur du bassin). Cette étude permet de classer comparativement l'érodabilité des bassins versants en 5 classes définies par pondération des variables précédentes.

Elle montre que les zones potentielles de forte production sédimentaire sont :

- le haut bassin versant de l'Arly contrôlé par le barrage des Mottets,

- le haut bassin versant de l'Isère (Isère, Sassière, Versoyen) contrôlé par les barrages de Tignes et d'Aigueblanche,

- le haut bassin versant de l'Arc (Arc, Ruisseau du Doron) contrôlé hors période de crue par les barrages du Freney, Pont des Chèvres et St Martin,

- les affluents de rive gauche de l'Arc moyen (Valloirette, Arvan, Glandon....).

De même, la faible érodabilité des massifs cristallins (Beaufortin, affluents de l'Isère moyenne, affluent de l'Arc inférieur) est nettement mise en évidence.

\subsubsection{Processus liés aux précipitations liquides}

Les précipitations interviennent de plusieurs manières dans le processus global d'érosion. Tout d'abord par l'effet "splash ", elles détachent le sédiment sur place et ce proportionnellement à l'énergie des précipitations. Ensuite, lorsque le ruissellement commence, elles transportent le sédiment vers le fond des talwegs. Enfin, le ruissellement induit lui-même une érosion ( «rill erosion »). A l'échelle du BV de l'Isère, ce processus dépend fortement des situations météorologiques et du couple (érosivité des pluies/érodabilité des terrains).

L'analyse des concentrations à Grenoble en relation avec les précipitations montre que les situations météorologiques engendrant de fortes valeurs de MeS sont :

- les retours d'Est ou Lombarde associés à des flux de SudSud Est avec une dépression centrée en Méditerranée. Ces flux sont accompagnés de précipitations violentes, avec fort effet orographique. Les précipitations sont particulièrement abondantes sur le haut Arc et sur les affluents rive gauche de l'Arc moyen soit l'ensemble des zones de forte érodabilité,

- les orages isolés sur les zones de forte érodabilité. Ces événements localisés sont cependant très difficiles à identifier selon le positionnement des cellules convectives par rapport au réseau pluviographique.

Les autres événements pluvieux type front d'Ouest donnent des précipitations plutôt sur l'Isère moyenne (Chartreuse, Belledonne). Ces précipitations sur des bassins à faible érodabilité donnent un débit élevé mais des concentrations variables selon le stock disponible (Exemple le $25 / 2 / 95$; Q Isère $>550 \mathrm{~m}^{3} / \mathrm{s}$ et $\mathrm{MeS}=1,5 \mathrm{~g} / \mathrm{l}$ ).

\subsubsection{Processus liés à la fusion nivale}

La période de fusion nivale génère également une érosion avec alternance de cycles de gel et de dégel du terrain superficiel, transport vers le fond des talwegs et érosion par le ruissellement. Ce processus est beaucoup plus homogène que le précédent car il est conditionné en grande partie par la température de l'air.

La fusion nivale dépend également du stock de neige, de sa répartition en altitude et de la structure du manteau neigeux. Bien évidemment les précipitations liquides sur le manteau neigeux jouent un grand rôle sur l'évolution de ce dernier, mais cette synergie de processus reste à ce jour très difficile à modéliser. En général les modèles se contentent de sommer les deux processus.

Un modèle de prévision des débits de l'Isère à Pont Royal en période de fusion a été mis au point par EDF/DTG [6]. Ce modèle de type degré.jour s'appuie sur la somme des températures des 5 derniers jours à Bonneval sur Arc (fig. 2).

\subsubsection{Rôle des débits}

La hauteur d'eau dans la rivière conditionne largement la capacité de transport de la rivière et notamment les phénomènes de reprises: la contrainte de cisaillement $\tau$ exercée par l'écoulement sur le fond est proportionnelle à la hauteur d'eau. De ce fait, de nombreuses formules empiriques relient la concentration en MES avec la hauteur d'eau [7].

Des différentes stations de mesures testées, c'est la hauteur d'eau mesurée dans l'Arc à Pont de St Rémy qui apparaît le mieux corrélé avec les $\mathrm{MeS}$ à Grenoble (coefficient de corrélation $=0,7$ en relation $L n-L n)$.

\section{MODÈLE PROPOSÉ - PROCESSUS SIMULÉS - CALAGE}

\subsection{Principes du modèle}

Le modèle tente de simuler, en reproduisant les processus globaux à l'échelle du bassin versant, le flux sédimentaire à

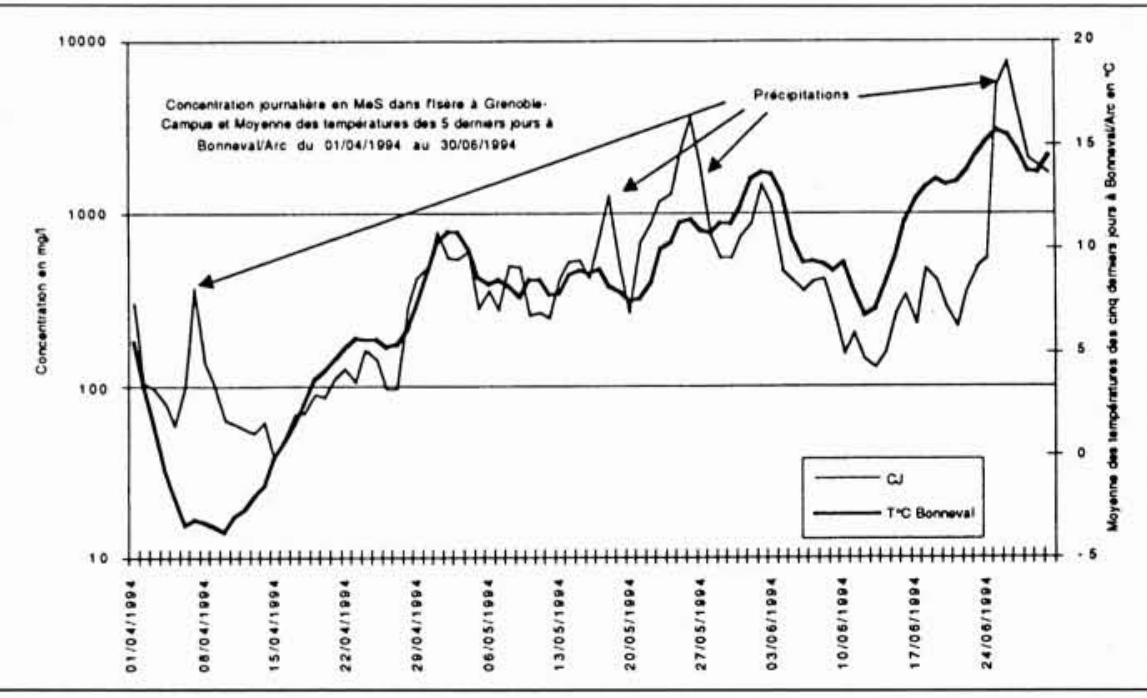

2. Relation entre Ln (MeS Grenoble) et $\mathrm{T}^{\circ} \mathrm{C}$ (Bonneval) sur 5 jours.
Le graphe des températures à Bonneval et du Logarithme népérien de la concentration en MeS sur la période avril-juin montre une bonne covariation en dehors des périodes de précipitation. Cependant, les MeS semblent moins élevées en fin de période nivale (limitation par le stock disponible?). 
l'échelle journalière. Sa conception s'appuie sur celle des modèles hydrologiques à réservoir et notamment le modèle MORDOR [8]. Ce modèle est capable de simuler le fonctionnement d'un bassin versant de montagne en prenant en compte de manière globale l'ensemble des processus, à partir d'une mesure de précipitation spatiale et d'une température de l'air. Il intègre notamment les phénomènes de stockage nival puis de fonte printanière.

Le modèle de flux sédimentaire part du constat que quelques sous-bassins de 1'Arc Supérieur et des affluents rive gauche de l'Arc Moyen participent très fortement aux apports de sédiments fins. Ils seront donc les seuls pris en compte et les apports par les autres bassins versants seront négligés par hypothèse.

Le modèle proposé s'appuie sur les processus suivants :

- Les précipitations sur les sous-bassins à forte érodabilité des sols sont séparées en précipitations liquides et en précipitations neigeuses grâce à la connaissance de l'hypsométrie du bassin versant et des températures de l'air.

- Les précipitations liquides ont une érosivité estimée par la somme des carrés des pluies horaires. Les pluviographes doivent être pris séparément du fait de l'hétérogénéité des précipitations notamment pour les phénomènes convectifs. Une partie des sédiments érodés s'évacue dans la rivière avec le flux liquide, l'autre alimente un réservoir conceptuel de stockage.

- La perte de valeur en eau du manteau neigeux sur les bassins à forte érodabilité a également une certaine érosivité. Cette perte est lissée pour tenir compte d'une certaine rémanence du processus de fusion. De même que pour les pluies, une partie des sédiments érodés s'évacue dans la rivière avec le flux liquide généré par la fusion nivale, l'autre alimentant le réservoir conceptuel de stockage.

- Au-dessus d'une hauteur seuil, le réservoir de stockage est repris par la rivière, proportionnellement à la hauteur d'eau supérieure à ce seuil.

- A chaque pas de temps, le stock est augmenté des apports (pluie et fusion) et diminué des flux repris.

- Le flux résultant est donc la somme des contributions directes des précipitations, de la fusion et de la reprise des sédiments par la rivière. Il possède également une certaine rémanence traduisant le fait qu'une fois repris, les sédiments sont transportés jusqu'à retrouver les conditions de dépôt.

\subsection{Schématisation des processus-modèles}

Voir figure 3.

\subsection{Variables d'entrée}

Les pluviographes retenus pour le modèle sont ceux de Ste Marie de Cuines pour l'Arc Moyen et de Bonneval pour l'Arc Supérieur. L'apport de pluviographes supplémentaires a été testé mais s'est révélé très peu significatif. Le thermographe associé permettant de déterminer les précipitations liquides est celui de Bonneval/Arc, le gradient thermique ayant été pris à $0,6^{\circ} \mathrm{C} / 100 \mathrm{~m}$ en moyenne.

Les télénivomètres retenus sont ceux de Plan Séti sur l'Arc Supérieur, de Bissorte pour l'Arc Moyen et de Roselend pour l'Isère. La fusion étant un phénomène beaucoup plus continu que les précipitations, une moyenne spatiale a été calculée à partir de ces informations.
La hauteur d'eau retenue est celle de l'Arc à Pont de St Rémy parce qu'étant mieux corrélée avec les concentrations en MES à Grenoble que la hauteur d'eau dans l'Isère.

\subsection{Calage du modèle}

Le modèle est calé par la méthode de Newton avec dérivées centrées, le critère classique à minimiser étant défini comme la somme des écarts au carré entre les flux observés et les flux modélisés. Les journées correspondant à des événements particuliers comme les chasses sont éliminées du critère.

La difficulté de caler ce type de modèle à plusieurs paramètres sur des flux solides vient de la dissymétrie de la distribution et donc du poids très important de quelques événements. L'adaptation trop élevée du modèle à l'échantillon de calage risquant de limiter ses performances en simulation, le critère a été modifié pour prendre en compte d'autres phénomènes.

Le critère classique est donc additionné d'une composante liée à l'écart modèle/mesure sur le tonnage cumulé des sédiments (environ $1 / 10$ des écarts ${ }^{2}$ ) et d'une composante liée au réservoir initial (écart entre le réservoir initial et la moyenne des valeurs du réservoir à la même date pour les autres années avec un poids de $1 / 10$ des écarts ${ }^{2}$ ). Cette dernière composante permet d'éviter un stockage ou un déstockage inter-annuel dans le réservoir conceptuel, et une trop forte adaptation des paramètres du Stock à l'échantillon de calage.

Le calage est réalisé sur les années 1994 et 1995, le modèle étant testé sur 1996.

\section{V —RÉSULTATS}

\subsection{Principaux paramètres du modèle - Résultats}

Le tableau 3 donne les valeurs optimales des principaux paramètres au sens du critère précédemment défini.

Le pourcentage de variance expliquée, si on exclut les périodes de chasses, est de $75 \%(R=0,87)$. Il convient néanmoins de tempérer ce résultat assez encourageant car les principales journées de transport solide en suspension début juin 1995 ont été exclues puisqu'une chasse d'Aigueblanche a été réalisée sur cette crue (concentration moyenne en MES $=10 \mathrm{~g} / \mathrm{l}$ en sortie du barrage).

\subsection{Analyse des écarts entre mesures et modèle}

Les écarts entre modèle et mesures apparaissent plus élevés sur les périodes où ont été effectuées des chasses, notamment celles d'Aigueblanche sur l'Isère (les flux sur ces chasses sont estimés en moyenne entre 100000 et 300000 tonnes).

Les flux durant les périodes hivernales sont assez mal représentés car les pluies d'hiver sont traduites en neige sur les bassins de l'Arc, et aucune fusion nivale n'est associée. Cela suggère que les phénomènes de reprises soient prépondérants à cette période. On observe également durant ces périodes que les variations de hauteur d'eau dans l'Arc sont de deux natures : augmentation liée aux précipitations sur les basses vallées d'une part et turbinages d'hiver d'autre part. Les deux phéno-

Tableau 3. - Valeurs optimales des principaux paramètres.

\begin{tabular}{|c|c|c|c|c|c|c|c|c|}
\hline$\alpha$ & afus & bfus & bdir & a1 (Arc Sup) & a2 (Arc Moy) & adir & ho & $\beta$ \\
\hline 0,36 & $6,210^{-6}$ & 3,76 & 0,29 & 0,017 & 0,014 & 0,19 & 0,87 & 0,14 \\
\hline
\end{tabular}




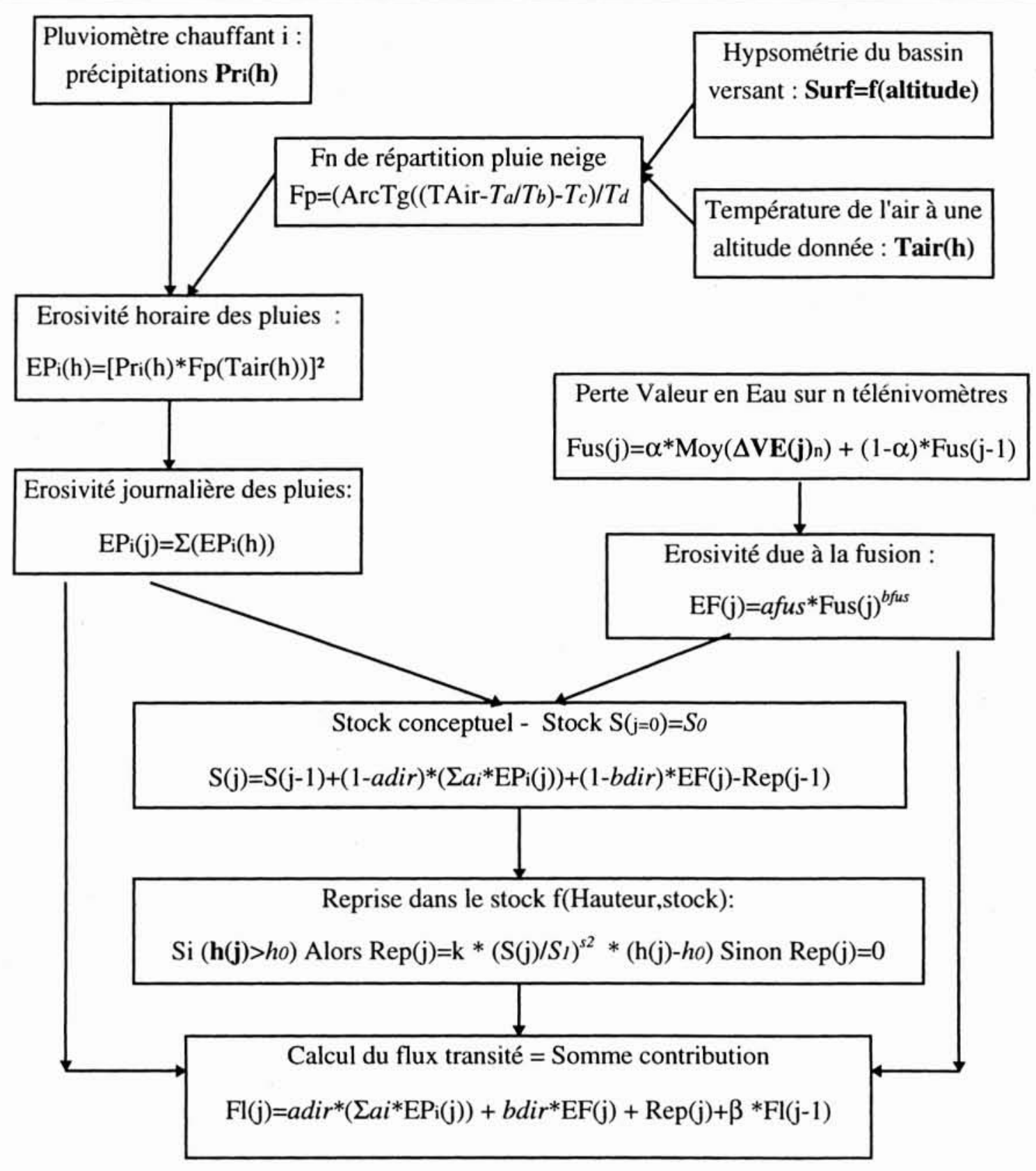

Nota : les variables d'entrées du modèle sont en gras ; les paramètres sont en italique

3. Schématisation des processus-modèle.

mènes n'ont pas du tout le même impact sur les flux sédimentaires, les turbinages n'entraînant que peu de flux.

Le rôle de l'influence anthropique sur les débits se retrouve également sur les faibles concentrations où les débits de l'Isère sont influencés par les manœuvres d'exploitation au Cheylas. Ces apports d'eau de l'Arc non pris en compte par le modèle et les reprises sédimentaires sur les variations de débit sont sans doute à l'origine de la difficulté à modéliser les faibles flux.

\subsection{Remarques sur les paramètres}

Le calage statistique du modèle fait apparaître que la fusion exprimée en $\mathrm{mm}$ doit être élevée à une puissance entre 3 et 
4. Comparaison chronologique entre mesures et modèle.

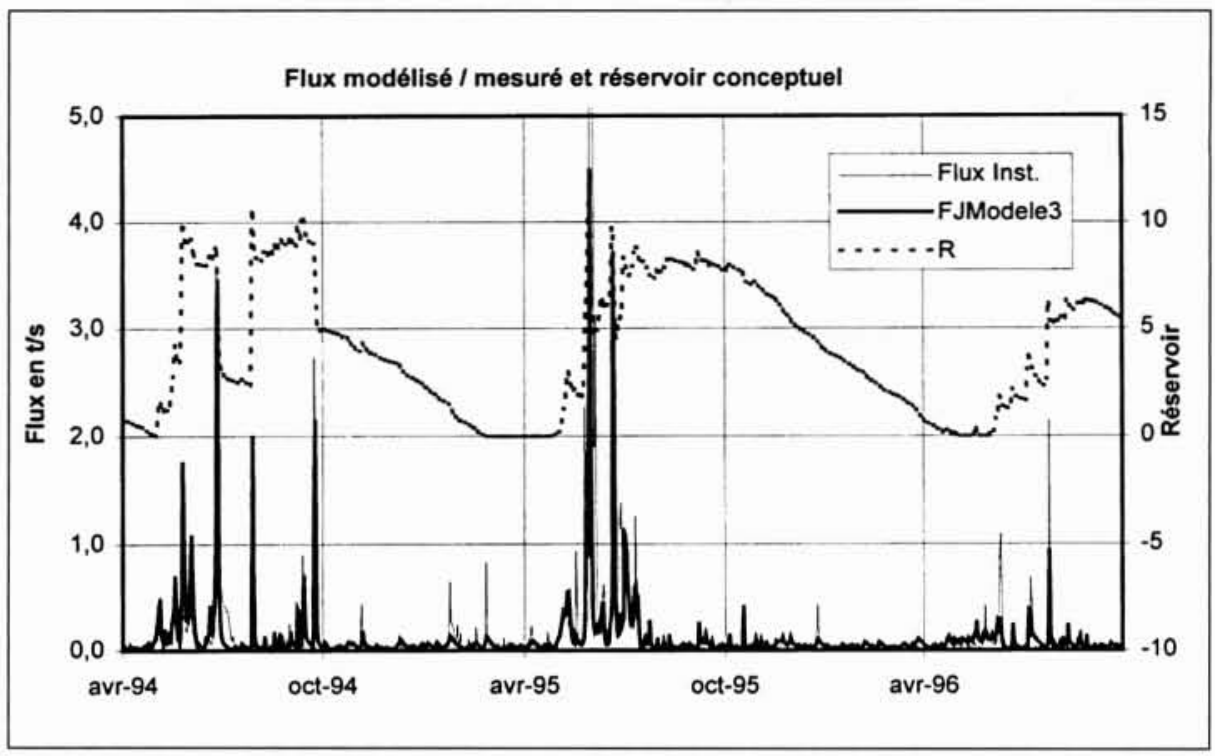

5. Comparaison entre mesure et modèle exceptées les périodes de chasses.

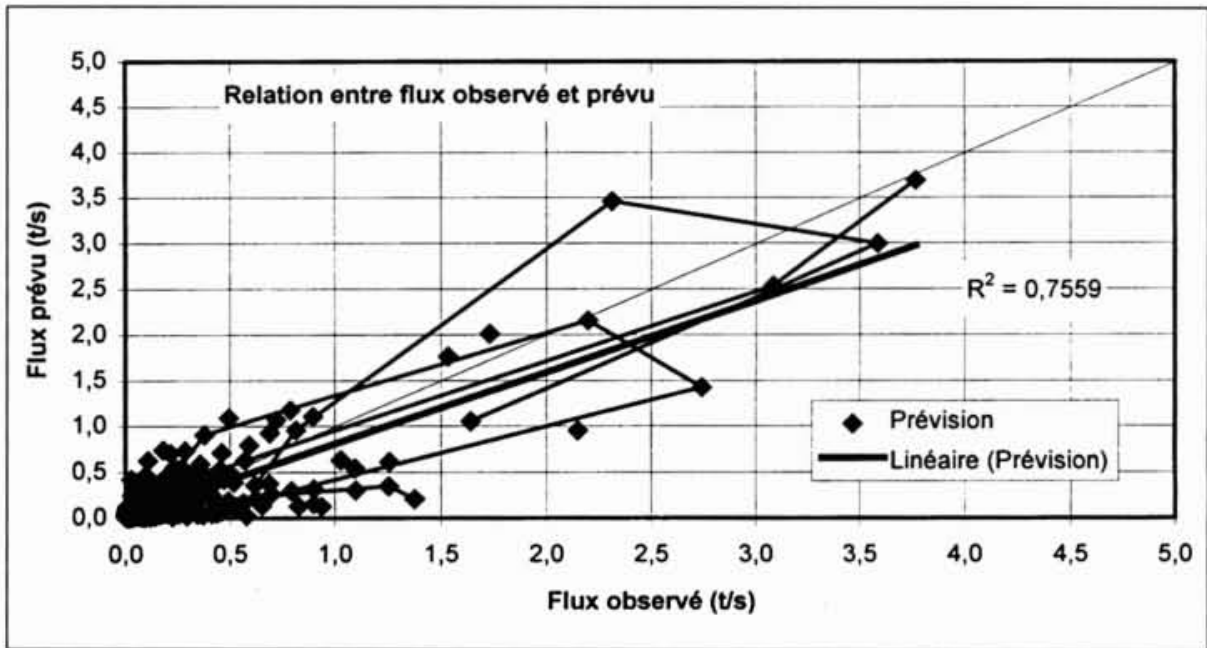

4 pour se traduire en érosivité. Cet exposant est nettement plus élevé que pour les précipitations où il a été pris à 2 d'après les données de la littérature (un essai de calage a donné environ 2,1). Cela peut sans doute s'expliquer par le fait que la fusion est un phénomène beaucoup plus généralisé que les précipitations orageuses, qu'elle s'accompagne généralement de cycles de gel/dégel et que les terrains sont exempts de végétation à la période de fusion.

Cette érosivité de la fusion se trouve également dans le coefficient de passage direct dans le flux puisque environ $1 / 3$ des flux générés par la fusion se retrouve très rapidement à Grenoble contre $1 / 5$ pour les pluies.

\section{DDISCUSSION}

Le modèle à réservoirs utilisé répond de manière satisfaisante aux objectifs initiaux, à savoir de permettre l'évaluation des flux journaliers de MeS à Grenoble à partir d'informations hydrométéorologiques collectées en temps réel sur le bassin versant. Il prend bien en compte la fusion et l'action des précipitations liquides. Le pas de temps choisi est un peu grand par rapport aux principaux phénomènes si bien que des déca- lages chronologiques peuvent apparaître selon que les précipitations sont plus ou moins tôt dans la journée.

L'ajout du module de production sédimentaire par fusion nivale semble incontournable, même si une démarche plus classique par modèle de fusion (degré.jour par exemple) pourrait être envisagée.

De même, la prise en compte de l'abattement nival dans le calcul de l'érosivité (calcul des précipitations liquides) apporte beaucoup au modèle. Reste cependant le problème délicat des précipitations sur manteau neigeux qui pour l'instant a été estimé simplement en sommant les deux effets.

Les améliorations du modèle viendront sans doute plus du module de transport que du module de production sédimentaire. Le fait d'ajouter une hauteur d'eau sur l'Isère pour tenir compte des reprises de stock sédimentaire dans le lit de l'Isère en amont immédiat de Grenoble semble prometteur. L'adjonction d'un modèle hydrologique permettant de reconstituer les tendances hors turbinages des débits de l'Arc pourrait également permettre de limiter les perturbations introduites dans le modèle par l'influence anthropique sur les débits.

Enfin se pose le problème de la stationnarité de la série chronologique de TSS compte tenu de toutes les modifications passées et actuelles du lit de la plupart des rivières alpines - et particulièrement de l'Arc sur les dernières années. 


\section{REMERCIEMENTS}

Je remercie vivement J.L.Peyri du Laboratoire de la Montagne Alpine pour avoir initié une série biquotidienne de mesures de matières en suspension à Grenoble et m'avoir transmis ses données. Ce type de série est très (trop ?) rare aujourd'hui et apparaît très riche d'enseignements après seulement trois années de mesures. Mes remerciements également à A. Gautheron pour s'être attaqué avec rigueur et ténacité à ce difficile problème qu'est la modélisation des flux de MES au cours de son DEA à l'ENSHMG.

\section{RÉFÉRENCES}

[1] CRAVERo J.M., Guichon P. (1989). - Exploitation des retenues et transport des sédiments. La Houille Blanche $\mathrm{N}^{\circ} 3 / 4$, pp. 292 295.

[2] Rambaud J., Clair M., Sandret J., Frau J.P., Khalanski M., RICARD C. (1988). - Expérience acquise dans les vidanges de retenues par Electricité de France et la Compagnie Nationale du Rhône. Commission internationale des grands barrages. Seizième Congrès des Grands Barrages, San Francisco.

[3] Callmail X., Poirel A., Lallement C., Clair M. (1993) Expérience acquise à EDF en matière de chasses de retenues. Modélisation du transit des MES sur l'aval. Colloque SHF. Comité technique Session n 148.

[4] Bouchard J.P., MAUREL F. (1995). - Estimation de l'entrainement des sédiments lors des vidanges de retenues. Rapport EDF DER HE 43/95.008/A.

[5] LATULIPPE C. (1995). - Recherches sur les zones de production sédimentaire et les transports de MES du bassin versant de l'Isère en amont de Grenoble. Mémoire de Maîtrise, Université J. Fourrier, Grenoble.

[6] Phienboupha (1977). - Analyse des débits de l'Isère. Essai de prévision par modèle statistique. Mémoire de Doctorat, Université J. Fourier.
[7] VAN Run (1993). - Principles of sedimentation in rivers, estuaries and coastal seas. Aqua Publication Amsterdam.

[8] GARçon R. (1996). - Prévision opérationelle des apports de la Durance à Serre Ponçon à l'aide du modèle MORDOR. Bilan de l'année 1994-1995. La Houille Blanche n 5/1996, pp. 71-76.

[9] Borges A.L.O. (1993). - Modélisation de l'érosion sur deux bassins versants expérimentaux des Alpes du Sud. Thèse de Doctorat, Université J. Fourier.

[10] BRUK S. (1986). - Méthodes de calcul de la sédimentation dans les lacs et réservoirs. Contribution au programme hydrologique international PHI-II A261.

[11] Danloux J., Laganier R. (1993). - Classification et quantification des phénomènes d'érosion, de transport et de sédimentation sur les bassins touchés par l'exploitation minière en Nouvelle Calédonie. Hydrologie continentale, Vol. $6, \mathrm{~N}^{*} 1$, pp. 15-28.

[12] GAFREJ R. (1993). - Modélisation conceptuelle du transfert des matières en suspension. Effets d'échelles spatio-temporelles. Thèse de Doctorat, Université Paris VI.

[13] GaUtheron A. (1995). - Elaboration d'un modèle explicatif des flux de MeS dans l'Isère à Grenoble. ENSHMG. Rapport de DEA.

[14] Kerth R., Cooley R. (1980). - Erosivity values for individual design storms. J. of Irrigation and Drainage Division $\mathrm{N}^{\circ} 137$, pp. 135-145.

[15] N'Guyen T.D., Lee S.H., Bois P., Tormos E., Giuliani Y. (1993). - Modélisation numérique du transport solide en écoulement non-permanent. Application à la retenue de Belley (Rhône). Colloque SHF - Comité technique, Session n ${ }^{\circ} 148$, 18 nov. 1993. Transports solides en eaux continentales.

[16] Meybeck M., Pasco A., Ragu A. (1994), - Evaluation des flux polluants dans les rivières : pourquoi, à quel prix? Etude Inter Agence $n^{\circ} 28$.

[17] PeYRI J.L. (1996). - Mesures des MES en Isère. Atelier SHF Transports Solides, Lyon.

[18] PolRel A. (1995). - Retour d'expérience sur 5 années d'application de la consigne de chasse sur la Basse Isère. Rapport EDF VID0082. 